\title{
Molecular Imaging of Inducible VEGF Expression and Tumor Progression in a Breast Cancer Model
}

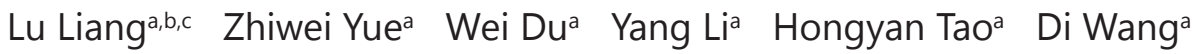 \\ Ran Wang ${ }^{a}$ Ziwei Huang ${ }^{a}$ Ningning He ${ }^{a}$ Xiaoyan Xie ${ }^{d}$ Zhongchao Han ${ }^{b, c} \quad \mathrm{Na} \mathrm{Liu}^{\mathrm{a}}$ \\ Zongjin Li ${ }^{\mathrm{a}, \mathrm{e}}$
}

aNankai University School of Medicine, Tianjin; ${ }^{b B e i j i n g ~ I n s t i t u t e ~ o f ~ H e a l t h ~ a n d ~ S t e m ~ C e l l s, ~ H e a l t h ~}$ \& Biotech Co., Beijing; ' National Engineering Research Center of Cell Products, Tianjin; ${ }^{\mathrm{d}} \mathrm{Cells}$ and Regenerative Medicine Laboratory, Beijing Institute of Transfusion Medicine, Beijing; 'The Key Laboratory of Bioactive Materials, Ministry of Education, Nankai University, the College of Life Science, Tianjin, China

\section{Key Words}

Molecular imaging - Bioluminescence - Vascular endothelial growth factor (VEGF) - Breast cancer • Angiogenesis

\begin{abstract}
Background: Tumor derived vascular endothelial growth factor (VEGF) can stimulate proliferation and migration of endothelial cells and recruit endothelial progenitor cells into tumors for vascular formation via a paracrine manner. Now increasing evidence suggests that VEGF also serves as an autocrine factor promoting cell survival and tumor angiogenesis. Real time visualization of VEGF activity in the early stages of tumor formation using molecular imaging will provide unprecedented insight into the biological processes of cancer. Methods: The mouse breast cancer cell line 4T1 was transfected with an inducible, bidirectional tetracycline (Bi-Tet) promoter driving VEGF and renilla luciferase (Rluc). This was used to quantitatively image conditional switching of VEGF by bioluminescence imaging (BLI) under the control of systemic administration of doxycycline. Simultaneously, 4T1 cells were labelled with the double fusion reporter gene (Fluc-eGFP) to establish a breast cancer model. Results: We found that inducible VEGF could promote proliferation and attenuate apoptosis due to oxidative stress in an autocrine manner in vitro. In vivo studies revealed that induction of VEGF expression during early tumor development not only dramatically enhanced tumor growth but also increased tumor angiogenesis as visualized by BLI. Finally, immunohistochemistry staining confirmed that inducing VEGF expression promoted cell survival and tumor neovascularization. Conclusion: Together the inducible bidirectional tetracycline (Bi-Tet) coexpression system combined with the dual bioluminescence imaging (BLI) system provides a platform to investigate a target gene's role in the pathologic process of cancer and facilitates noninvasive monitoring of biological responses in real time.

L. Liang and Z. Yue contributed equally to this work.




\section{Cellular Physiology Cell Physiol Biochem 2017;42:407-415

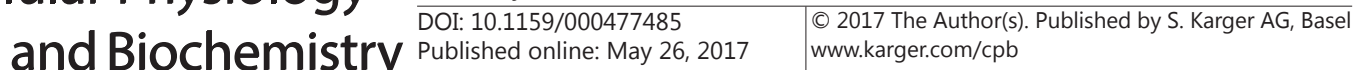

Liang et al.: Imaging of VEGF Expression and Tumor Progression

\section{Introduction}

Tumor angiogenesis is an essential process involving the formation of new blood vessels and plays a critical role in tumor growth and survival $[1,2]$. Without new blood vessels to transport oxygen and nutrients, tumor growth cannot progress beyond 1-2 $\mathrm{mm}^{3}$ [2]. Secretion of angiogenic growth factors such as vascular endothelial growth factor (VEGF) to recruit endothelial progenitor cells from pre-existing blood vessels (angiogenesis) allows tumors to overcome this growth limitation. VEGF secreted by tumor cells can also promote tumor angiogenesis and growth by acting as an autocrine survival factor for tumor cells [24]. Blocking the expression of VEGF could alleviate the progression of cancer [5], as well as tumor metastasis [6, 7]. However, studying VEGF in tumor progression has proved challenging due to the lack of an available method to non-invasively monitor tumor angiogenesis and responses in vivo. Conventional methods to monitor tumor development usually requires the sacrifice of many animals at multiple time points making them inconvenient, timeconsuming and unrepeatable [8].

Molecular imaging is defined as the visualization, characterization, and quantification of biological processes in living organisms at the cellular and molecular level by means of non-invasive imaging [8]. Bioluminescence imaging (BLI) of luciferase reporter genes, as a powerful research strategy, has been used to monitor tumor growth and regression $[3,8,9]$. More importantly than this, two or more luciferase reporters with biochemical distinction can provide an opportunity to monitor different biological processes, such as exploration of the correlation between key protein activity and relative physiological states, tumor volume in tumor development [10], or evaluation of therapeutic efficacy in the same animal simultaneously and successively $[3,11]$.

VEGF is mainly located in the extracellular matrix, resulting in high concentration in the tumor microenvironment. Non-invasive assessment of the association of VEGF-level in the microenvironment with breast cancer can predict and monitor the response of tumor cells in development.

The characteristics of propagations and metastases of the 4T1 tumor in Balb/c mice is very similar to that of human mammary cancer [12]. In this study, we transduced 4T1 mouse breast cancer cells with firefly luciferase (Fluc) and an inducible, bidirectional tetracycline (BiTet) promoter driving VEGF165 and renilla luciferase (Rluc). Dual bioluminescence imaging (BLI) was applied to monitor VEGF expression by Rluc imaging and tumor progression by Fluc imaging respectively. Moreover, VEGF induced angiogenesis was tracked in a transgenic mouse model.

\section{Methods and Materials}

\section{Cell lines and cell culture}

Murine breast cancer 4T1 cells were cultured in RPMI 1640 medium (Invitrogen, Carlsbad, CA) supplemented with 10\% FBS (Biological Industries, Israel), 1\% non-essential amino acids (NEAA) and $1 \%$ penicillin/streptomycin. For tracking transplanted cells, 4T1 cells were transduced with a lentiviral vector carrying firefly luciferase (Fluc) and enhanced green fluorescence protein (eGFP) double fusion (DF) reporter gene as described previously $[3,13,14]$. Stable 4T1-DF eGFP cells were sorted by fluorescenceactivated cell sorter (FACS, BD Biosciense). Afterward, Fluc activity within different cell numbers was confirmed ex vivo using Xenogen IVIS Lumina II system (Xenogen, Alameda, CA) as described [15].

Preparation of Bi-Tet cell lines

The bidirectional tetracycline (Bi-Tet) co-expression system, both human VEGF165 and renilla luciferase (Rluc) in a bidirectional cassette was constructed as described previously [4]. Briefly, the regulator plasmid pTet-On (Clontech, Mountain View, CA) was transfected into 4T1 or 4T1-DF cells. Afterward, the response plasmid Bi-Tet was then co-transfected into 4T1 or 4T1-DF cells with a selection plasmid at a ratio of $4: 1$. The cells transfected only with the regulator plasmid pTet-on were used as control. 


\section{Cellular Physiology Cell Physiol Biochem 2017;42:407-415 \begin{tabular}{l|l} 
and Biochemistry Published online:May 26, 2017 & $\begin{array}{l}\text { (c) } 2017 \text { The Author(s). Published by S. Karger AG, Basel } \\
\text { www.karger.com/cpb }\end{array}$ \\
\hline
\end{tabular}}

Liang et al.: Imaging of VEGF Expression and Tumor Progression

Cell proliferation and apoptosis assays

To determine the optimal dose-response relationship between doxycycline and Rluc/VEGF165 production, increasing dosages of doxycycline up to $2 \mu \mathrm{g} / \mathrm{ml}$ were applied. As a positive correlation exists between Fluc activity and cell number, bioluminescence imaging (BLI) was employed to measure cell proliferation at 24,48 , and 72 hours after treatment with doxycycline. To investigate whether VEGF could rescue hydrogen peroxide $\left(\mathrm{H}_{2} \mathrm{O}_{2}\right)$ induced injury, $1 \times 10^{5}$ cells per well were seeded in 24 wells and subcultured with doxycycline for 48 hours. $\mathrm{H}_{2} \mathrm{O}_{2}$ was added at a concentration of $500 \mu \mathrm{M}$ and incubated for 12 hours. BLI was performed to evaluate cell survival. $2 \times 10^{5}$ cells per well were seeded in 6 wells and same treated by doxycycline and $\mathrm{H}_{2} \mathrm{O}_{2}$, then stained with an Annexin V-EGFP apoptosis detection kit (KeyGen Biotech, China) according to the manufacturer's instructions.

\section{Real-Time PCR}

Total RNA was extracted with TRIzol reagent (Invitrogen, Grand Island, NY) following the manufacturer's instructions. cDNAs was synthesized with oligo (dT) as primers using the First-strand cDNA Synthesis System (TransGen Biotech, China). Real-time PCR analysis using TransStart Green qPCR SuperMix Kit (TransGen Biotech, China) was performed on Opticon ${ }^{\circledR}$ System (Bio-Rad, Hercules, CA). Relative mRNA folding changes were identified with $2^{-\Delta c t}$ method.

\section{Tumor model}

Balb/c mice were obtained from Laboratory Animal Center, the Academy of Military Medical Sciences (Beijing, China). Transgenic mice, which expressed firefly luciferase (Fluc) under the promoter of vascular endothelial growth factor receptor 2 (vegfr2-luc) [11], were obtained from Xenogen Corporation (Hopkinto, MA, USA). Animal protocols were approved by the Nankai University Animal Care and Use Committee, which conform to the Guide for the Care and Use of Laboratory Animals published by the US National Institutes of Health (8th edition, 2011). To establish a breast tumor model with Balb/c mice 1x10 $10^{5}$ Bi-Tet 4T1-DF cells and control 4T-1 DF cells were subcutaneously injected beneath both sides of the mammary gland fat pads of 8-week-old female Balb/c mice. Mice were administered $0.5 \mathrm{mg} / \mathrm{ml}$ doxycycline in drinking water for the first 7 days, then changed to normal water until sacrificed. Doxycycline-inducible expression of renilla luciferase (Rluc) was determined by BLI. To monitor in vivo angiogenesis in real time, tumor models were set up with transgenic vegfr2-luc mice and Bi-Tet 4T1 cells. All mice were sacrificed at the end of the experiments, and tumor tissues from each group were harvested for further analysis.

\section{Optical bioluminescence imaging}

Bioluminescence imaging (BLI) was performed using IVIS Lumina II system (Xenogen Corporation, Hopkinton, MA) as described [15]. Briefly, tumor-bearing mice were administered with D-luciferin (150 $\mathrm{mg} / \mathrm{kg}$, Biosynth International, Naperville, IL) by intraperitoneal injection for Fluc imaging to track tumor progression and with coelenterazine $(2.5 \mathrm{mg} / \mathrm{kg}$; NanoLight Technology, Pinetop, AZ) by intravenous injection for Rluc imaging to evaluate the expression of VEGF. This dual luciferase imaging approach was well established for tracking tumor progression and simultaneously monitoring VEGF expression in vivo in this study.

\section{Histological analysis}

For histologic assessment, tumors were embedded in OCT compound (Miles Scientific). Frozen sections ( $5 \mu \mathrm{m}$ thick) were processed for immunostaining with an antibody against CD31 (BD Pharmingen, San Jose, CA). And the nuclei were stained using 4, 6-diamidino-2-phenylindole (DAPI, BD Pharmingen) as counterstaining.

\section{Statistical analysis}

All data are expressed as means \pm SEM. An independent t-test was used for two-group comparisons, and one-way ANOVA for multiple group comparisons, with suitable post hoc analysis. Significance difference was defined as $P<0.05$. 


\section{Cellular Physiology Cell Physiol Biochem 2017;42:407-415 \begin{tabular}{l|l} 
and Biochemistry & DOI: 10.1159/000477485 \\
Published online: May 26, 2017 & $\begin{array}{l}\text { C } 2017 \text { The Author(s). Published by S. Karger AG, Basel } \\
\text { www.karger.com/cpb }\end{array}$
\end{tabular}

\section{Results}

\section{Labeling of $4 T 1$ cells with DF reporter genes}

To quantitatively evaluate cell survival of 4T1s stably transfected with Bi-Tet when exposed to doxycycline a double fusion reporter gene construct (Fluc-eGFP) was transduced into the cells. Positive eGFP cells were sorted by FACS (Fig. 1A) and immunofluorescence assay revealed robust expression of eGFP. A strong correlation between Fluc activity and cell numbers was observed in 4T1 cells by the Xenogen IVIS system (Fig. 1B), which demonstrated the ability to assess cell numbers in vitro and tumor growth in vivo by analyzing Fluc signal intensity. The Fluc activity correlated linearly with cell number $\left(\mathrm{R}^{2}=0.99\right)$ as shown in Fig. $1 \mathrm{C}$ and $\mathrm{D}$.

\section{Inducible VEGF and renilla luciferase (Rluc) expression}

To track VEGF expression in real time in breast cancer cells using this molecular imaging strategy, 4T1 cells expressing inducible, bidirectional VEGF and Rluc were generated. Highlevel bidirectional expression of VEGF and Rluc was obtained in the presence of doxycycline, which was absent in those cells cultured without doxycycline. As revealed in Fig. 2A, the expression of VEGF and Rluc was highly dependent on the presence of doxycycline.

Dose-response analysis was performed on $4 \mathrm{~T} 1$ cells in order to optimize their dosing response to doxycycline. Transfected $4 \mathrm{~T} 1$ cells were treating with $0,0.1,0.2,0.5,2 \mu \mathrm{g} / \mathrm{ml}$ doxycycline. Quantitative measurements indicated that $0.5 \mu \mathrm{g} / \mathrm{mL}$ is the ideal doxycycline concentration for peak production of VEGF and Rluc (Fig. 2B and C). The correlative analysis between VEGF and Rluc revealed that doxycycline can tightly regulate the expression these two different genes via the Bi-Tet system (Fig. 2D). In addition, we found VEGF secreted from cancer cells was highly upregulated by doxycycline, as well as HIF-1 $\alpha$, Ang- 1 and FGF2 (Fig. 2E).

\section{Induction of VEGF in 4T1cells confers pro-survival effects}

Tumor cells can secrete VEGF in a paracrine manner to stimulate endothelial progenitor cell angiogenesis. It has also been reported that VEGF is an autocrine survival/protective factor for several tumors [16-19]. In this study, accurate assessment of tumor response to VEGF was observed by molecular imaging. In vitro increased cell proliferation activity of the Bi-Tet 4T1-DF cells were observed at time points 48- and 72-h (Fig. 3A). These results were consistent with cell counting analysis. To evaluate the pro-survival effects of VEGF, Bi-Tet

Fig. 1. Generation of 4T1-DF cells Labeling of cells with double fusion reporter genes. (A) Schema of lentiviral construct showing ubiquitin promoter driving Fluc and eGFP. (B) Brightfield and fluorescence microscopy showing robust eGFP expression in undifferentiated $4 \mathrm{~T} 1$ cells. (C, D) BLI of $4 \mathrm{~T} 1$ cells on 6 -well plate shows a robust correlation between cell numbers and Fluc activity.

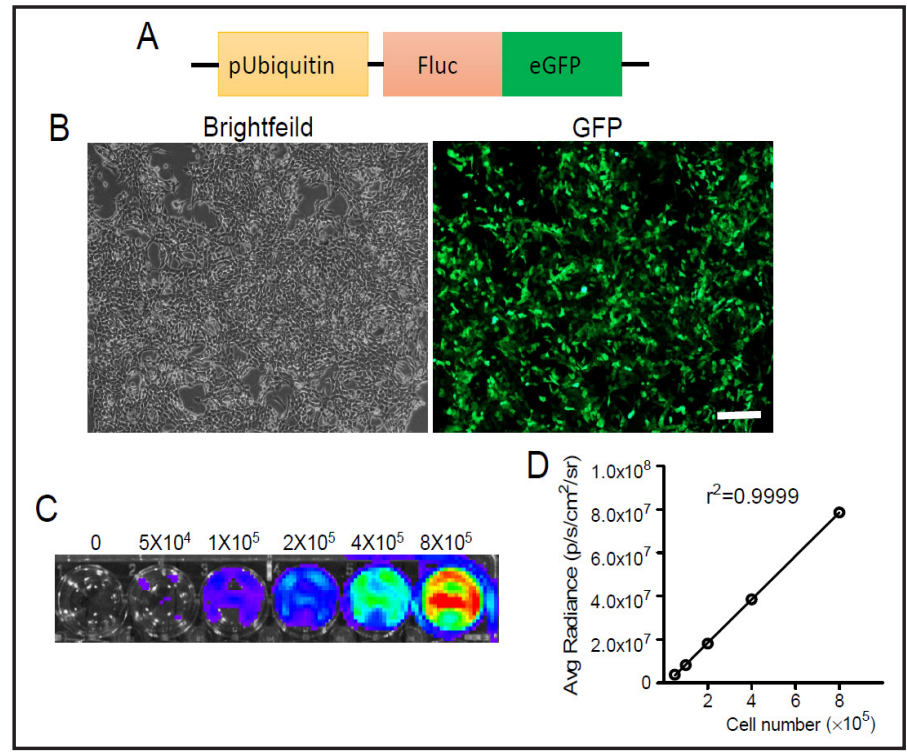




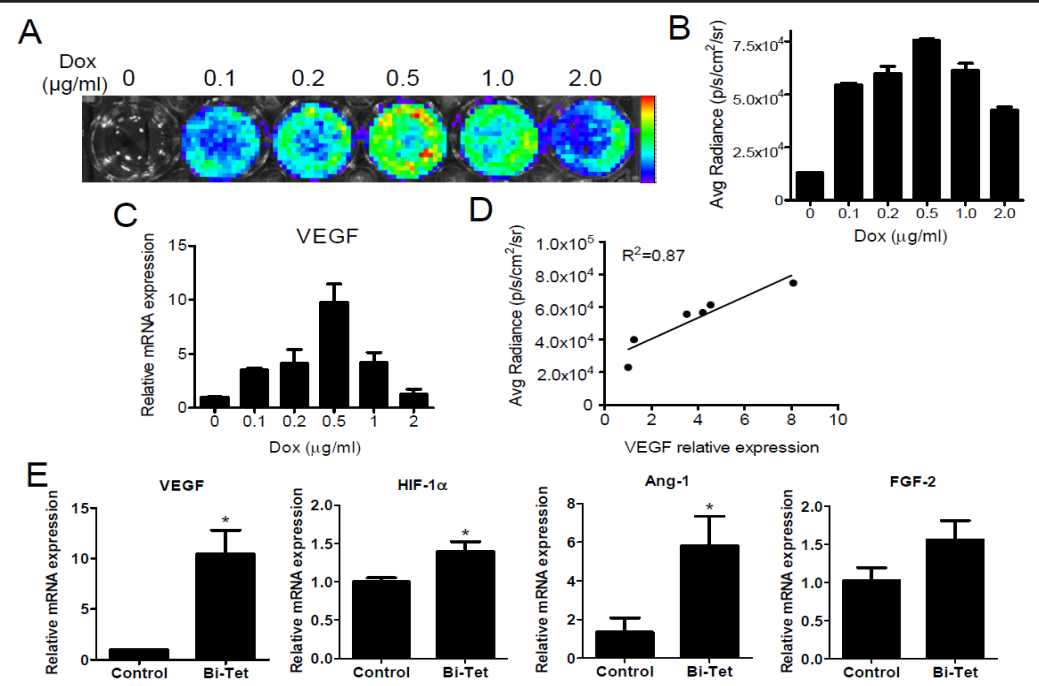

Fig. 2. Generation of 4T1 cells with bidirectionally expressing VEGF and Rluc. (A) Expression of VEGF and Rluc induced by doxycycline; optimal dosage of doxycycline determined to be $0.5 \mu \mathrm{g} / \mathrm{ml}$. (B) Quantitative analysis of Rluc signals. (C) Real-time RT-PCR showing peak production of VEGF at $0.5 \mu \mathrm{g} / \mathrm{ml}$ of doxycycline. (D) Robust correlation between the expression of VEGF and Rluc. (E) Up-regulation of VEGF, HIF-1 $\alpha$, Ang-1 and FGF2 secreted from cancer cells with doxycycline administration.
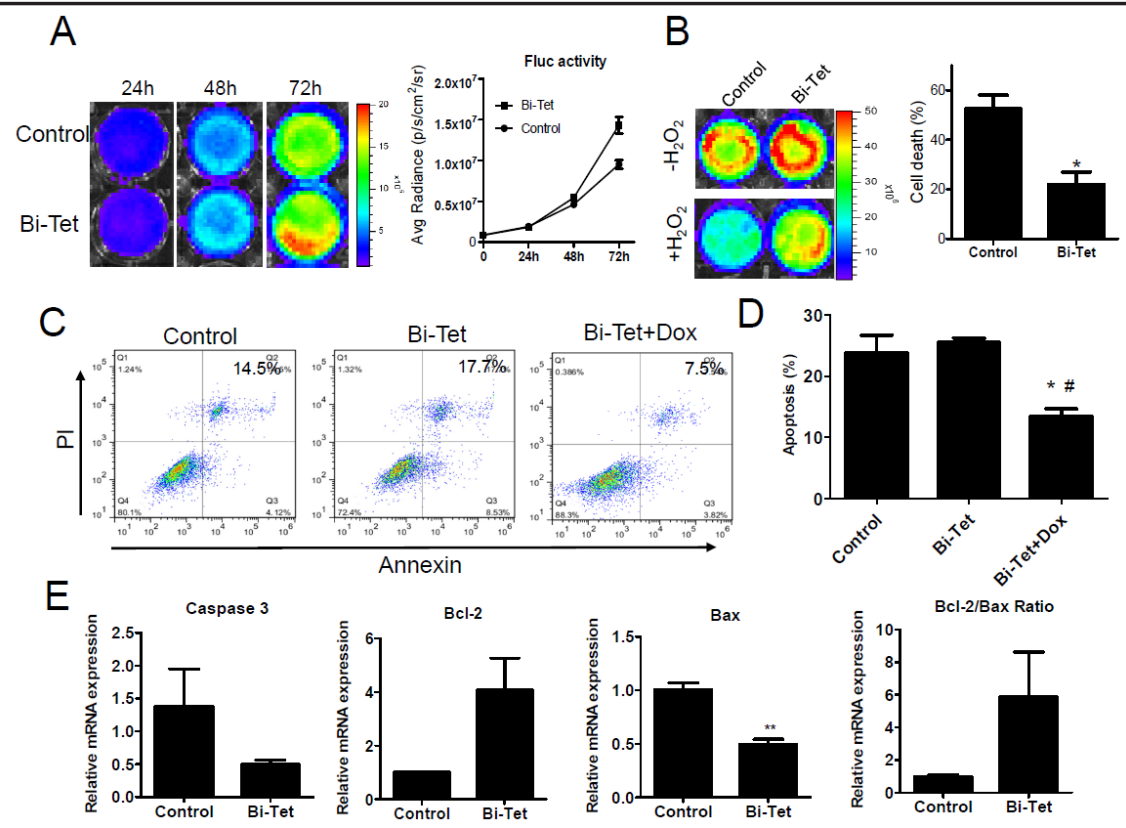

Fig. 3. Assessment of cell characteristics after Dox induction in vitro. (A)Increased in vitro cell proliferation activity of Bi-Tet 4T1-DF cells compared with control 4T1-DF cells at 48- and 72-h. (B) Bi-Tet 4T1-DF cells and control cells treated with doxycycline for 48 hours. Oxidative damage was then induced by adding $\mathrm{H}_{2} \mathrm{O}_{2}$ solution $(500 \mu \mathrm{M})$ and cell survival was determined by BLI to evaluate the pro-survival effects of VEGF. The ability of proliferation and against oxidative stress was upregulated in Bi-Tet 4T1-DF group. (C) Oxidative stress damage was induced by $\mathrm{H}_{2} \mathrm{O}_{2}$ solution $(500 \mu \mathrm{M})$ and cell apoptosis was determined by FACS to evaluate the anti-apoptosis effect of VEGF. Inducible VEGF could attenuate apoptosis due to oxidative stress. (D) Quantitative analysis shows the ability of anti-apoptosis was upregulated in Bi-Tet 4T1-DF group after Dox induction (*, compared with Control $P<0.05$; \#, compared with Bi-Tet $P<0.05$ ). (E) Lower expression level of caspase- 3 and Bax with a high ratio of bcl-2; consistent with our hypothesis that VEGF promotes the tumor growth. 

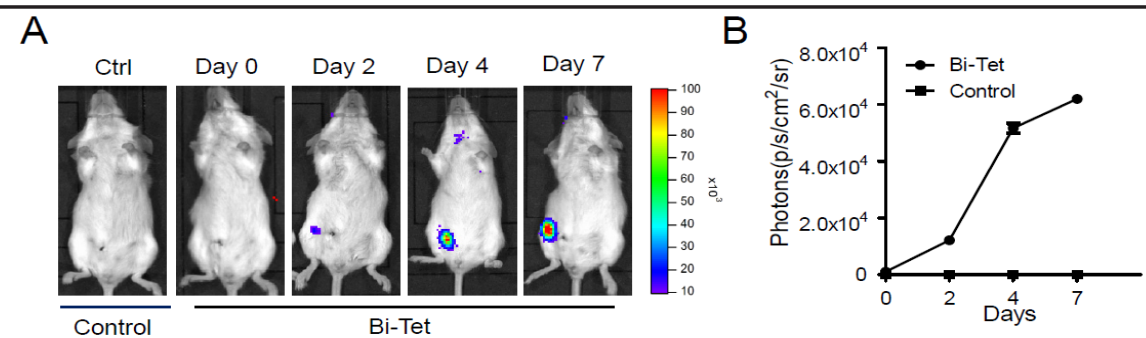

C
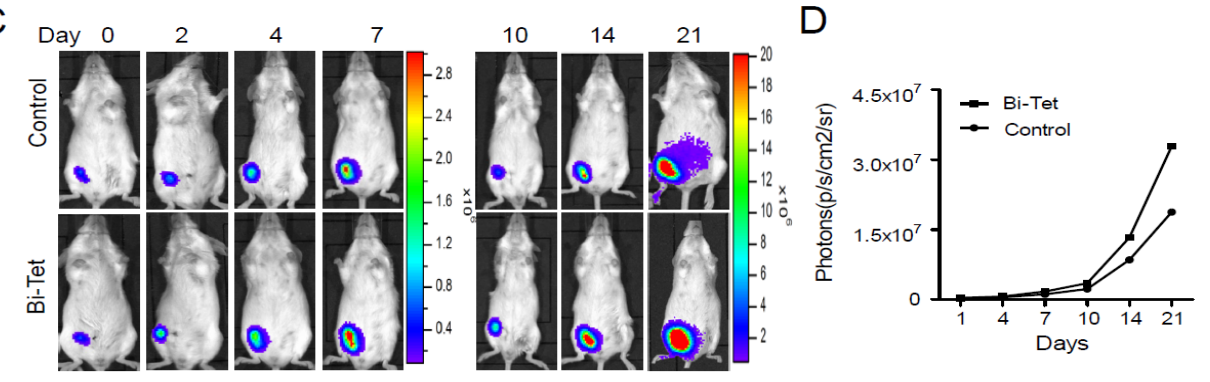

Fig. 4. BLI of tumor growth and inducible VEGF expression. (A) Rluc signals obtained at the second day of doxycycline induction; rapid increase of Rluc signal in the Bi-Tet group in early tumor development. (B) Quantitative measurements showing a progressive increase of Rluc signals in Bi-Tet group. (C) Fluc imaging showing a significant increase in tumor growth with doxycycline induction. (D) Quantitative analyses of both groups.

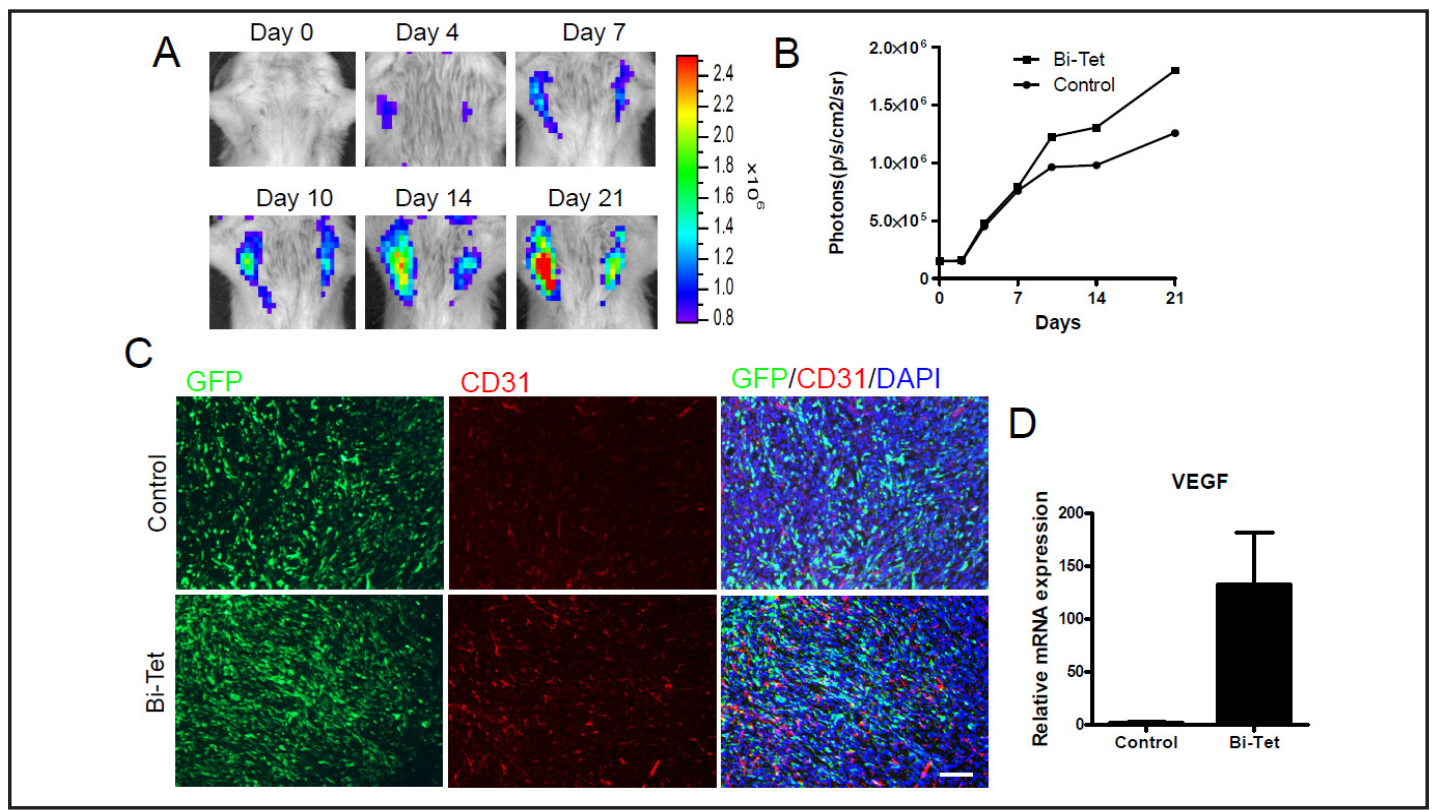

Fig. 5. Analysis of tumor angiogenesis. (A, B) Observation of Fluc signals observed in Bi-Tet group from day 4. High levels of VEGF may initiate angiogenic switching during tumorigenesis. Signal intensity increased more significantly in Bi-Tet group. (C, D) Increased angiogenesis observed in the Bi-Tet group by CD31 immunofluorescence staining and real time PCR.

4T1-DF cells and control 4T1-DF cells were treated with doxycycline for 48 hours. Oxidative damage was then induced by adding $\mathrm{H}_{2} \mathrm{O}_{2}(500 \mu \mathrm{M})$ for 12 hours and cell survival was determined by BLI and FACS. Bi-Tet 4T1-DF cells had significantly lower rates of apoptosis/ death compared with control cells (Fig. 3B, C\&D). The expression of caspase-3 and the ratio of bcl-2 to Bax were consistent with these results (Fig. 3E).

\section{KARGER}




\section{Cellular Physiology Cell Physiol Biochem 2017;42:407-415 \\ \begin{tabular}{l|l} 
DOI: 10.1159/000477485 & O 2017 The Author(s). Published by S. Karger AG, Basel \\
www.karger.com/cpb
\end{tabular} \\ Liang et al.: Imaging of VEGF Expression and Tumor Progression}

Effects of inducible VEGF on tumor growth

To determine the effects of VEGF on early stage breast cancer progression and development Fluc imaging was introduced. We injected $5 \times 10^{5} \mathrm{Bi}$-Tet 4T1-DF cells and control 4T1-DF cells into Balb/c mice and doxycycline $(0.5 \mathrm{mg} / \mathrm{ml})$ was administered via drinking water for 7 days. Doxycycline-induced VEGF expression was imagined by Rluc. Rluc signals were obtained from the second day of doxycycline administration (Fig. 4A\&B). Meanwhile, Fluc imaging showed a significant increase in the tumor growth rate with doxycycline administration in the Bi-Tet 4T1-DF group compared to the 4T1-DF group (Fig. 4C\&D).

\section{Bioluminescent imaging of tumor angiogenesis}

To further explore whether the effects of VEGF on tumor cells in promoting early tumor growth is associated with angiogenesis, the imaging of angiogenesis was performed within the vegfr2-luc mice tumor model. Here we found that the Fluc signals were observed in Bi-Tet group from day 4 , and signal intensity increased most significantly and immediately following a period of tumor growth (Fig. 5A\&B). Furthermore, microvascular density analysis results revealed enhanced angiogenesis by CD31 staining in the Bi-Tet 4T1-DF group (Fig. 5C). In addition, the enhanced expression of VEGF was remarkably increased in tumor tissue of BiTet 4T1-DF group when analyzed by real-time PCR (Fig. 5D).

\section{Discussion}

In this present study, we set up a dual BLI model to simultaneously monitor VEGF and tumor progression using imaging. We demonstrated that the mouse breast cancer cell line 4T1 could be stably transfected with an inducible bidirectional promoter vector containing both VEGF and Rluc, in which the expression of Rluc and VEGF level is well correlated with doxycycline administration. The response of $4 \mathrm{~T} 1$ cells labeled by a double-fusion reporter gene (Fluc-eGFP) to VEGF can then be noninvasively tracked by dual BLI in vivo.

To investigate the development process, mechanism and therapeutic response of cancer by real time imaging is crucial for evaluating new molecular targets, as well as for the development of new drugs for translational medicine [8, 20]. Molecular imaging approaches, especially bioluminescence imaging (BLI), provide a highly sensitive and versatile tool for investigating cancer biology and therapy. As the substrates for Fluc and Rluc are D-luciferin and coelenterazine respectively, it is possible to monitor two molecular processes in the same animal simultaneously. Visualizing mechanisms of action of anti-cancer drugs therapeutic effects and treatment efficacy in vivo in real time BLI will provide a versatile platform for cancer research. This approach is sensitive and even quantitative. However, optimization is still needed as the algorithms used to reconstructing 3D fluorescence image are still prone to error [21].

Previous studies demonstrated VEGF plays a key role in tumor growth $[6,22]$. Therefore, we injected 4T1 cells into live animals and expression of VEGF was induced by systemic administration of doxycycline. This resulted in a high concentration of VEGF in the tumor microenvironment which can be visualized with BLI. Our results revealed that the role of VEGF derived from tumor cells in promoting proliferation and combating tumor microenvironment stress was partly responsible for cell survival and early tumor formation. VEGF deficiency severely disrupted both angiogenic switching and tumor growth [23], whereas high levels of VEGF can initiate neovasculature formation during tumorigenesis. In this study, the differences in angiogenic ability between the Bi-Tet group and control group were observed in vegfr2-luc mice. Several studies have demonstrated that VEGF can create a perivascular niche for cancer stem cells (CSCs) by promoting angiogenesis and that VEGF directly affects CSCs through Nrp1 in an autocrine loop to stimulate cancer stemness and renewal [24]. This promotion of CSCs by VEGF may be the mechanism for earlier tumor initiation in our results. 


\section{Cellular Physiology Cell Physiol Biochem 2017;42:407-415 \begin{tabular}{l|l} 
and Biochemistry Published online:1May 26, 2017 & $\begin{array}{l}\text { (c) } 2017 \text { The Author(s). Published by S. Karger AG, Basel } \\
\text { www.karger.com/cpb }\end{array}$
\end{tabular}}

Liang et al.: Imaging of VEGF Expression and Tumor Progression

In conclusion, we developed a mouse model for directly visualizing the comprehensive effects of induced-VEGF secretion on enhancing tumor cell survival and angiogenesis via the dual BLI imaging technique. VEGF clearly promotes early tumor formation, and consequently, further affects subsequent tumor development. Our results indicate that expression of VEGF not only dramatically enhanced tumor growth but also increased the rate of early tumor formation. Taken together, the inducible bidirectional tetracycline (Bi-Tet) co-expression system combined with dual BLI provides a versatile tool for the noninvasive monitoring of biological processes and responses in cancer research.

\section{Acknowledgment}

This work was partially supported by grants from the National Natural Science Foundation of China (81671734, 81371620, 81320108014), Fundamental Research Funds for the Central Universities (63171335), and Program for Changjiang Scholars and Innovative Research Team in University (IRT13023). The authors thank Maura Hannon from Cork, Ireland, for her English editing of the article.

\section{Disclosure Statement}

The authors declare that there is no conflict of interest regarding the publication of this paper.

\section{References}

1 Folkman J: What is the evidence that tumors are angiogenesis dependent? J Natl Cancer Inst 1990;82:4-6.

2 Zhao Q Li Z: Angiogenesis. Biomed Res Int 2015;2015:135861.

- 3 Leng L, Wang Y, He N, Wang D, Zhao Q, Feng G, Su W, Xu Y, Han Z, Kong D, Cheng Z, Xiang R, Li Z: Molecular imaging for assessment of mesenchymal stem cells mediated breast cancer therapy. Biomaterials 2014;35:5162-5170.

4 Xie X, Cao F, Sheikh AY, Li Z, Connolly AJ, Pei X, Li RK, Robbins RC, Wu JC: Genetic modification of embryonic stem cells with VEGF enhances cell survival and improves cardiac function. Cloning Stem Cells 2007;9:549563.

5 Zhang W, Zou C, Pan L, Xu Y, Qi W, Ma G, Hou Y, Jiang P: MicroRNA-140-5p inhibits the progression of colorectal cancer by targeting VEGFA. Cell Physiol Biochem 2015;37:1123-1133.

-6 Huang S, He P, Peng X, Li J, Xu D, Tang Y: Pristimerin Inhibits Prostate Cancer Bone Metastasis by Targeting PC-3 Stem Cell Characteristics and VEGF-Induced Vasculogenesis of BM-EPCs. Cell Physiol Biochem 2015;37:253-268.

7 Xu L, Sun K, Xia M, Li X, Lu Y: MMP13 Regulates Aggressiveness of Pediatric Multiple Myeloma Through VEGF-C. Cell Physiol Biochem 2015;36:509-516.

-8 Wang L, Wang Y, Li Z: Nanoparticle-based tumor theranostics with molecular imaging. Curr Pharm Biotechnol 2013;14:683-692.

-9 Su W, Wang L, Zhou M, Liu Z, Hu S, Tong L, Liu Y, Fan Y, Kong D, Zheng Y, Han Z, Wu JC, Xiang R, Li Z: Human embryonic stem cell-derived endothelial cells as cellular delivery vehicles for treatment of metastatic breast cancer. Cell Transplant 2013;22:2079-2090.

10 Wang L, Su W, Liu Z, Zhou M, Chen S, Chen Y, Lu D, Liu Y, Fan Y, Zheng Y, Han Z, Kong D, Wu JC, Xiang R, Li Z: CD44 antibody-targeted liposomal nanoparticles for molecular imaging and therapy of hepatocellular carcinoma. Biomaterials 2012;33:5107-5114. 


\section{Cellular Physiology Cell Physiol Biochem 2017;42:407-415

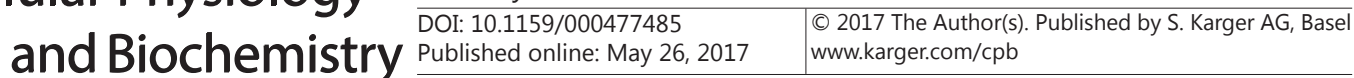

Liang et al.: Imaging of VEGF Expression and Tumor Progression

11 Feng G, Zhang J, Li Y, Nie Y, Zhu D, Wang R, Liu J, Gao J, Liu N, He N, Du W, Tao H, Che Y, Xu Y, Kong D, Zhao Q, Li Z: IGF-1 C domain-modified hydrogel enhances cell therapy for AKI. J Am Soc Nephrol 2016;27:23572369.

-12 Baklaushev VP, Grinenko NF, Yusubalieva GM, Abakumov MA, Gubskii IL, Cherepanov SA, Kashparov IA, Burenkov MS, Rabinovich EZ, Ivanova NV, Antonova OM, Chekhonin VP: Modeling and integral X-ray, optical, and MRI visualization of multiorgan metastases of orthotopic 4T1 breast carcinoma in BALB/c mice. Bull Exp Biol Med 2015;158:581-588.

13 Zhou M, Wang L, Su W, Tong L, Liu Y, Fan Y, Luo N, Zheng Y, Zhao H, Xiang R, Li Z: Assessment of therapeutic efficacy of liposomal nanoparticles mediated gene delivery by molecular imaging for cancer therapy. J Biomed Nanotechnol 2012;8:742-750.

14 Zhao Q Tong L, He N, Feng G, Leng L, Sun W, Xu Y, Wang Y, Xiang R, Li Z: IFN-gamma mediates graft-versusbreast cancer effects via enhancing cytotoxic T lymphocyte activity. Exp Ther Med 2014;8:347-354.

15 He N, Feng G, Li Y, Xu Y, Xie X, Wang H, Wang Y, Ou L, Pei X, Liu N, Li Z: Embryonic stem cell preconditioned microenvironment suppresses tumorigenic properties in breast cancer. Stem Cell Res Ther 2016;7:95.

16 Harmey JH, Bouchier-Hayes D: Vascular endothelial growth factor (VEGF), a survival factor for tumour cells: implications for anti-angiogenic therapy. Bioessays 2002;24:280-283.

17 Bachelder RE, Crago A, Chung J, Wendt MA, Shaw LM, Robinson G, Mercurio AM: Vascular endothelial growth factor is an autocrine survival factor for neuropilin-expressing breast carcinoma cells. Cancer Res 2001;61:5736-5740.

18 Barr MP, Bouchier-Hayes DJ, Harmey JH: Vascular endothelial growth factor is an autocrine survival factor for breast tumour cells under hypoxia. Int J Oncol 2008;32:41-48.

19 Masood R, Cai J, Zheng T, Smith DL, Hinton DR, Gill PS: Vascular endothelial growth factor (VEGF) is an autocrine growth factor for VEGF receptor-positive human tumors. Blood 2001;98:1904-1913.

-20 Paulmurugan R: Real Time Dynamic Imaging and Current Targeted Therapies in the War on Cancer: A New Paradigm. Theranostics 2013;3:437-447.

21 Blow N: In vivo molecular imaging: the inside job. Nature Methods 2009;6:465-469.

22 Cai H, Xue Y, Li Z, Hu Y, Wang Z, Liu W, Li Z, Liu Y: Roundabout4 Suppresses Glioma-Induced Endothelial Cell Proliferation, Migration and Tube Formation in Vitro by Inhibiting VEGR2-Mediated PI3K/AKT and FAK Signaling Pathways. Cell Physiol Biochem 2015;35:1689-1705.

-23 Inoue M, Hager JH, Ferrara N, Gerber HP, Hanahan D: VEGF-A has a critical, nonredundant role in angiogenic switching and pancreatic beta cell carcinogenesis. Cancer Cell 2002;1:193-202.

24 Beck B, Driessens G, Goossens S, Youssef KK, Kuchnio A, Caauwe A, Sotiropoulou PA, Loges S, Lapouge G, Candi A, Mascre G, Drogat B, Dekoninck S, Haigh JJ, Carmeliet P, Blanpain C: A vascular niche and a VEGFNrp1 loop regulate the initiation and stemness of skin tumours. Nature 2011;478:399-403. 